\title{
THE EFFECT OF TEXTURE ON GRAIN GROWTH \\ Theoretical Consideration of Grain Growth in the Presence of Textures
}

\author{
X. B. ZHAO \\ Department of Materials Science and Engineering, Zhejiang University, \\ Hangzhou, P.R. China
}

(Received 25 July 1992)

\begin{abstract}
Based on the analysis of grain boundary energy change during the migration of grain boundaries a statistic model of grain growth was developed in which both specific energy and mobility of a grain boundary section were considered to be dependent on the grain orientations in both sides of the boundary. It is shown that the textural effects, i.e. the anisotropy of grain boundary energy and mobility, on the process of grain growth can be described quantitatively with the integrated partial diffusivities of each texture component. It is found that the anisotropy of boundary energy causes an increase on the critical grain size for the minor texture component and a decrease for the major component. This leads to a texture strengthening, i.e. the increase of the volume percentage of the major texture component, before a strong texture change occurs. The model predictions agree with the experimental results well.
\end{abstract}

KEY WORDS Grain growth, texture, grain boundary.

\section{INTRODUCTION}

For the grain growth (GG) in the presence of textures, the specific energy $\gamma$ and mobility $m$ of various grain boundaries (GBs) are different due to the anisotropy of GBs. Generally, a small angle GB has much smaller values of $\gamma$ and $m$ than those of a large angle GB. Such anisotropy of GBs inhibits the process of normal GG, leads to a change of the kinetics of GG and may result in a marked abnormal GG (secondary recrystallization) if the textures are strong enough.

Theoretical treatments of normal GG in 2-dimensional case have been given by J. von Neumann (1952). By integrating around the grain perimeter he found that the growth rate of a grain's area, $A$, can be specified by the number of sides, $N$, with:

$$
\frac{d A}{d t}=\frac{\pi}{3} M \gamma(N-6)
$$

Assuming that the average GB curvature could be represented by $\left(1 / R_{\mathrm{cr}}\right)-$ $(1 / R)$, with $R$ being the grain size defined by the radius of the volume-equal sphere:

$$
R=\left(\frac{3 V}{4 \pi}\right)^{1 / 3}
$$


and $R_{\text {cr }}$ being a system constant called critical radius, M. Hillert (1965) gave a statistic model of normal GG in 3-dimensional case with the growth rate of:

$$
\frac{d R}{d t}=m \gamma\left(\frac{1}{R_{\mathrm{cr}}}-\frac{1}{R}\right)
$$

Based on Hillert-model and taking into account anisotropic $\gamma$ and $m$, recently, G. Abbruzzese, K. Lücke and $\mathbf{H}$. Eichelkraut derived a statistical model (AL-model) for the quantitative analysis of the effect of textures on GG. The growth rate of grains with orientation $H$ and size $R_{i}$ is given as follows:

$$
\left(\frac{d R}{d t}\right)_{i}^{H}=\tilde{M}^{H}\left(\frac{1}{\tilde{R}_{c}^{H}}-\frac{1}{R_{i}}\right)
$$

where $\tilde{M}^{H}$ and $\tilde{R}_{c}^{H}$ first defined by (Abbruzzese and Lücke, 1986; Eichelkraut, Abruzzese and Lücke, 1988):

$$
\left\{\begin{array}{l}
\tilde{M}^{H}=2 \sum_{K} \sum_{j} \varphi_{j}^{K} R_{j}^{2} m^{H K} \bar{\gamma} / \sum_{K} \sum_{j} \varphi_{j}^{K} R_{j}^{2} \\
\tilde{R}_{c}^{H}=\sum_{K} \sum_{j} \varphi_{j}^{K} R_{j}^{2} m^{H K} / \sum_{K} \sum_{j} \varphi_{j}^{K} R_{j} m^{H K}
\end{array}\right.
$$

and later modified to (Abbruzzese, Lücke and Eichelkraut, 1988):

$$
\left\{\begin{array}{l}
\tilde{M}^{H}=\sum_{K} \sum_{j} \varphi_{j}^{K} R_{j}^{2} m^{H K} \bar{\gamma}^{H} / \sum_{K} \sum_{j} \varphi_{j}^{K} R_{j}^{2} \\
\tilde{R}_{c}^{H}=\sum_{K} \sum_{j} \varphi_{j}^{K} R_{j}^{2} m^{H K} \bar{\gamma}^{H} / \sum_{K} \sum_{j} \varphi_{j}^{K} R_{j} m^{H K} \bar{\gamma}^{K}
\end{array}\right.
$$

where $\varphi_{j}^{K}$ is the grain size distribution function defined as the grain number fraction of the orientation-size class $[K, j], m^{H K}$ and $\gamma^{H K}$ are the mobility and the specific boundary energy of the $H K$ boundary section between a $H$-oriented grain and a $K$-oriented neighbouring grain respectively, $\bar{\gamma}^{K}$ is the average energy of all $K$-oriented grains and $\bar{\gamma}$ the average specific boundary energy of the whole structure.

A different treatment of GG had been used by $\mathrm{H}$. J. Bunge and E. Dahlem (1987, 1988). For a considered grain $v$ with orientation $H$, the boundaries which surround grain $v$ are called here "surrounding boundaries" and all boundaries between the neighbouring grains of $v$ (e.g. the $\zeta-\mu$ and $\mu-\eta$ boundary in Figure 1) are called "radial boundaries." Thus, the balance of grain boundary energy of the surrounding boundaries and the radial boundaries determines the growth or shrinkage of the considered grain. Assuming that all surrounding boundaries of $H$-oriented grains have the same specific boundary energy of $\bar{\gamma}^{H}$ and mobility of $\bar{m}^{H}$, and all radial boundaries have the same energy of $\bar{\gamma}$, a two-dimensional grain growth model has been developed by Bunge and Dahlem (1987):

with

$$
\left(\frac{d R}{d t}\right)_{i}^{H}=\tilde{M}^{H}\left(\frac{1}{\tilde{R}_{\mathrm{cr}}^{H}}-\frac{1}{R_{i}}\right)
$$

$$
\tilde{M}^{H}=\bar{m}^{H}\left(\bar{\gamma}^{H}-\frac{3}{2 \pi} \bar{\gamma}\right), \quad \tilde{R}_{\mathrm{cr}}^{H}=\frac{2 \pi}{2.5}\left(\frac{\bar{\gamma}^{H}}{\bar{\gamma}}-\frac{3}{2 \pi}\right) \bar{R}
$$




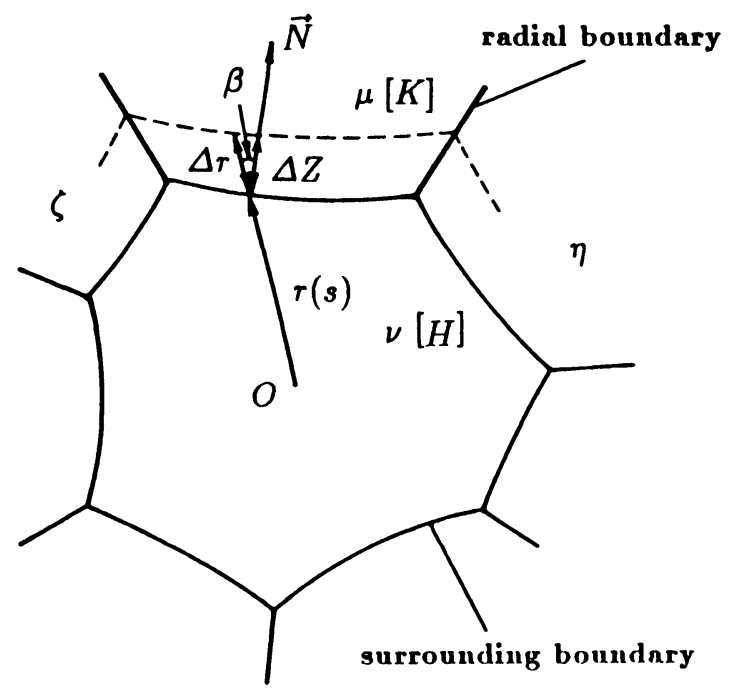

Figure 1 Description of grain boundaries.

It is well known that the driving force of the migration of a curved boundary section is directed towards the centre of curvature of the boundary section with the value on unit area of

$$
P=\gamma\left(\frac{1}{\rho_{1}}+\frac{1}{\rho_{2}}\right)
$$

with $\rho_{1}$ and $\rho_{2}$ being two principal curvature radii of the local GB. The migration rate of the boundary section is then given by

$$
v=m P=m \gamma\left(\frac{1}{\rho_{1}}+\frac{1}{\rho_{2}}\right)
$$

The growth rate of a grain is the aggregate effect of the local migration rates of all boundary sections of the grain. Since the local boundary curvatures $\rho_{1}$ and $\rho_{2}$ are practically unmeasurable, methods which need neither evaluation nor assumption of boundary curvatures should be practically better for the analysis of GG.

In the present work, a new statistic model for GG in the presence of textures will be developed using the method similar to Bunge's, i.e. based on the analysis of the changes of boundary energy. However, since both $m$ and $\gamma$ in Eq. (10) could be different from one boundary section to another even for the same grain, the grain growth rate will be calculated here by the average on the local migration rate, not on the mobility and specific energy.

\section{BASIC HYPOTHESES}

The following statistical postulates are used in the present work:

(1) The local equilibrium hypothesis. It is considered that the local equilibrium of GB holds at the intersect points and along the intersect lines. 
(2) The linear migration rate hypothesis. The local migration rate of a GB section is assumed to be linearly proportional to the local driving force acting on it, (cf. Equation (10)).

(3) The homogeneity hypothesis. It is supposed that all grains in a given orientation-size class have the same orientation, size and surroundings, and therefore the same growth rate in the statistic sense.

(4) The randomness hypothesis. It is assumed that the positions of grains in the structure are statistically random. This means that the boundary area of a $[H, i]$ grain in contact with $[K, j]$ grains can be given by

$$
S_{i j}^{H K}=S_{i} \frac{\varphi_{j}^{K} S_{j}}{\sum_{K, j} \varphi_{j}^{K} S_{j}}=S_{i} \frac{\varphi_{j}^{K} R_{j}^{2}}{\sum_{K, j} \varphi_{j}^{K} R_{j}^{2}}
$$

with $S_{i}$ and $S_{j}$ being the surface areas of $i$ - or $j$-sized grain respectively.

(5) The boundary similarity hypothesis. This means in the present work that any parameter about GBs that is invariant under a uniform magnification is also independent of time.

Here the first four postulates have been used by most researchers (e.g. Abbruzzese and Lücke, 1986) and the last one is included in the SSS (statistical self-similarity) hypothesis, which was suggested by W. W. Mullins (1986) and already proven by theoretical analyses, computer simulation results and direct experimental observations (for further details see overview given by Mullins and Viñals, 1989).

Two important conclusions can be deduced from the boundary similarity hypothesis: firstly, the change of the radial radius $r(s)$ at point $s$ on the boundary $S^{v \mu}$ of grain $v$ in contact with a neighbouring grain $\mu$, i.e. the change of the distance between point $s$ and the centre of grain $v$ in an infinite short time $\Delta t$, $\Delta r(s)$, is linearly proportional to $r(s)$ :

$$
\frac{\Delta r(s)}{r(s)}=\text { const. } \quad\left(s \in S^{v \mu}\right)
$$

and secondly, $S^{\nu \mu}$ is symmetrical about axis $\vec{Q}$, which goes through the grain centre and the centre of $S^{\nu \mu}$, i.e. $r(s)$ depends only on angle $\theta$ between the radial direction $\vec{R}$ and axis $\vec{Q}$, (cf. Fig. 2).

\section{The DERIVATION OF THE GRAIN GROWTH RATE}

For a grain $v$ in class $[H, i]$ we consider first a boundary section contacting with a neighbouring grain $\mu$ in class $[K, j]$ with boundary area $S^{\nu \mu}$, specific energy $\gamma^{H K}$ and mobility being $m^{H K}$. We assume that an infinitesimal boundary element $d s$ at point $s$ on the boundary section $S^{\nu \mu}$ moves outwards in the normal direction with $\Delta Z(s)=\Delta r(s) \cos \beta(s)$ (see Figure 1) in time $\Delta t$. According to Eq. (12) one has:

$$
\Delta Z(s)=\Delta r(s) \cos \beta(s) \propto r(s) \cos \beta(s)
$$

For the sake of simplicity, we define

$$
r^{*}(s)=r(s) \cos \beta(s)
$$




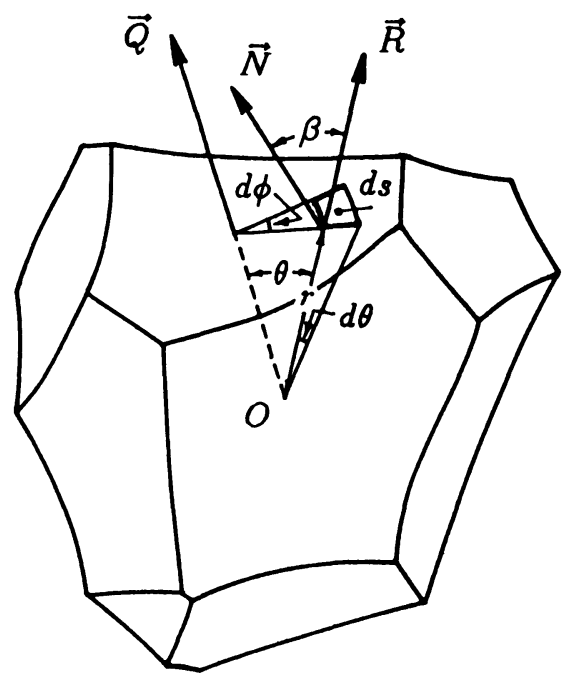

Figure 2 Angles related to an infinitesimal boundary element $d s$.

Therefore

$$
\Delta Z(s)=\overline{\Delta Z}^{v \mu} \frac{r^{*}(s)}{\overline{r^{*} v \mu}}
$$

with $\overline{\Delta Z}^{v \mu}$ and $\bar{r}^{* \mu}$ being the mean normal displacement and the mean projected length of radial radii on normal directions of the GB section $S^{\nu \mu}$. According to the boundary similarity hypothesis and Figure 2, the area $d s$ can be given by:

$$
d s=r(\theta) \sin \theta d \phi \cdot \frac{r(\theta) d \theta}{\cos \beta}
$$

Hence the change of $d s$ in time $\Delta t$ should be

$$
\Delta(d s)=\frac{\partial(d s)}{\partial r} \Delta r=2 r(\theta) \frac{\sin \theta}{\cos \beta} \Delta r d \phi d \theta
$$

Integrating it over $\theta$ and $\phi$ on $S^{\nu \mu}$ and using Eqs. (13), (14), (15) and (16) one has

$$
\begin{aligned}
\Delta S^{v \mu} & =2 \iint_{S^{\nu \mu}} r(\theta) \frac{\sin \theta}{\cos \beta} \Delta r d \phi d \theta \\
& =2 \frac{\frac{\Delta Z^{v \mu}}{\bar{r}^{* \mu}}}{\int} \iint_{S^{v \mu}} r^{2}(\theta) \frac{\sin \theta}{\cos \beta} d \phi d \theta \\
& =2 \frac{\overline{\Delta Z^{v \mu}}}{\overline{r^{*} \mu}} \cdot S^{v \mu}=C_{1} \overline{\Delta Z^{v \mu}} S^{v \mu} \frac{R^{v}}{S^{v}}
\end{aligned}
$$

with $C_{1}$ being defined as

$$
C_{1}=\frac{2 S^{v}}{\overline{r^{*} v \mu} R^{v}}
$$


It is clear that $C_{1}$ is a dimensionless factor depending on the grain shape. For spherical grains $C_{1}=8 \pi$ and in all other cases $C_{1}>8 \pi$. Therefore, the change of the surrounding boundary energy due to the migration of $S^{\nu \mu}$ in time $\Delta t$ is:

$$
\Delta E_{1}=\gamma^{H K} \Delta S^{\nu \mu}=C_{1} \gamma^{H K} \overline{\Delta Z^{\nu \mu}} S^{\nu \mu} \frac{R^{v}}{S^{\nu}}
$$

Meanwhile, the radial boundaries will be decreased due to the outward migration of $S^{\nu \mu}$ by area $\Delta S_{R}^{\nu \mu}$. This leads to a corresponding decrease of boundary energy by

$$
\Delta E_{2}^{v \mu}=-\bar{\gamma}^{K} \Delta S_{R}^{v \mu}
$$

where $\bar{\gamma}^{K}$ is used here as the statistical average value of the specific energies of the considered radial boundaries since the orientations of neighbours ( $\zeta$ and $\eta$ ) are indefinable at the moment. The change of the radial boundary can be given approximately by:

$$
\Delta S_{R}^{v \mu}=\frac{1}{2} L_{R}^{v \mu} \overline{\Delta R}_{R}^{v \mu}
$$

Here $L_{R}^{v \mu}$ is the perimeter of $S^{\nu \mu}$, and $\overline{\Delta R}_{R}^{v \mu}$ is the average displacement $L_{R}^{v \mu}$ in the

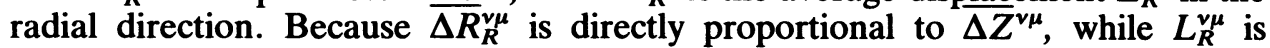
directly proportional to $S^{\nu \mu}$ and inversely proportional to the sizes of grain $v$ and $\mu, R^{v}$ and $R^{\mu}$, we have

$$
\Delta E_{2}=\frac{C_{2}}{2} \bar{\gamma}^{K} \overline{\Delta Z^{\nu \mu}} S^{\nu \mu}\left(\frac{1}{R^{v}}+\frac{1}{R^{\mu}}\right)
$$

with $C_{2}$ being again a geometrical factor.

The work done by the driving force on boundary section $S^{\nu \mu}$ in $\Delta t, W^{\nu \mu}$, can be represented either by the decrease of GB energy or by integrating the product of the local driving force $P(s)$ and the local boundary migration $\Delta Z(s)$ on $S^{\nu \mu}$ :

$$
W^{v \mu}=-\left(\Delta E_{1}^{v \mu}+\Delta E_{2}^{v \mu}\right)=\int_{S^{v \mu}} P(s) \Delta Z(s) d s
$$

Since $P(s)$ is proportional to $\Delta Z(s)$ we have:

$$
P(s)=\bar{P}^{v \mu} \frac{r^{*}(s)}{\overline{r^{*} v \mu}}
$$

with $\overline{\boldsymbol{P}}^{v \mu}$ being the average driving force defined by

$$
\bar{P}^{v \mu}=\frac{1}{S^{v \mu}} \int_{S^{\nu \mu}} P(s) d s
$$

Using Eqs. (15), (24) and (25), we have:

with

$$
W^{v \mu}=C_{3} \bar{P}^{v \mu} S^{v \mu} \overline{\Delta Z}^{v \mu}
$$

$$
C_{3}=\int_{s^{\nu \mu}}\left[\frac{r^{*}(s)}{\overline{r^{* v \mu}}}\right]^{2} d s
$$


being also a dimensionless factor which represents the variation of $r^{*}(s)$ in $S^{\nu \mu}$ and equals to unity if $S^{\nu \mu}$ is a spherical section with the centre at the grain centre, and some what larger than one in all other cases.

Inserting Eqs. (20), (23) and (27) into Eq. (24), we obtain

$$
\bar{P}^{v \mu}=\frac{C_{2}}{C_{3}} \bar{\gamma}^{K}\left(\frac{1}{R^{v}}+\frac{1}{R^{\mu}}\right)-\frac{C_{1}}{C_{3}} \frac{R^{v}}{S^{v}} \gamma^{H K}
$$

Here since both $C_{1}$ and $C_{3}$ are geometrical factors representing the deviations of grains from spheres, as a reasonable approximations we assume $C_{1} / C_{3}=8 \pi$. Defining furthermore $C^{\prime}=C_{2} / C_{3}$, the average driving force acting on $S^{v \mu}$ per area can be written as:

$$
\bar{P}^{v \mu}=C^{\prime} \bar{\gamma}^{K}\left(\frac{1}{R^{v}}+\frac{1}{R^{\mu}}\right)-8 \pi \frac{R^{v}}{S^{v}} \gamma^{H K}
$$

The volume change rate of grain $v$ caused by the migration of $S^{\nu \mu}$ is then given by

$$
\begin{aligned}
\frac{d V^{v \mu}}{d t} & =m^{H K} \int_{S^{v \mu}} P(s) d s=S^{v \mu} m^{H K} \bar{P}^{v \mu} \\
& =C^{\prime} m^{H K} \bar{\gamma}^{K} S^{v \mu}\left(\frac{1}{R^{v}}+\frac{1}{R^{\mu}}\right)-8 \pi m^{H K} \gamma^{H K} R^{v} \frac{S^{\nu \mu}}{S^{v}}
\end{aligned}
$$

Now we are considering not only the boundary between $v$ and $\mu$ but all boundaries between $[H, i]$-grains like $v$ and $[K, j]$-grains like $\mu$. Thus in the statistical sense the average boundary area between one $[H, i]$-grain and its $[K, j]$-neighbours being $S_{i j}^{H K}$ defined by Eq. (11). Using $S_{i j}^{H K}$ instead of $S^{\nu \mu}$ in Eq. (31) and summing it over $K$ and $j$ one obtains:

$$
\left(\frac{d V}{d t}\right)_{i}^{H}=C^{\prime} \sum_{K} \sum_{j} m^{H K} \bar{\gamma}^{K} S_{i j}^{H K}\left(\frac{1}{R_{i}}+\frac{1}{R_{j}}\right)-8 \pi \sum_{K} \sum_{j} m^{H K} \gamma^{H K} S_{i j}^{H K} \frac{R_{i}}{S_{i}}
$$

According to the definitions of $R$ and $S_{i j}^{H K}$, Eqs. (2) and (11), we have the average growth rate of grains in class $[H, i]$ :

$$
\left(\frac{d R}{d t}\right)_{i}^{H}=\frac{1}{4 \pi R_{i}^{2}}\left(\frac{d V}{d t}\right)_{i}^{H}=\frac{\tilde{G}_{1}^{H}}{\bar{R}}+\frac{\tilde{G}_{2}^{H}}{R_{i}}-2 \frac{\tilde{M}^{H}}{R_{i}}
$$

where the growth parameters $\tilde{G}_{1}^{H}, \tilde{G}_{2}^{H}$ and $\tilde{M}^{H}$ are called "partial diffusivities" since they have the same dimension of diffusivity, $\left[L^{2} T^{-1}\right]$, and are defined by

$$
\left\{\begin{array}{l}
\tilde{G}_{1}^{H}=C \sum_{K} \sum_{j} \varphi_{j}^{K} R_{j} \bar{R} m^{H K} \bar{\gamma}^{K} / \sum_{K} \sum_{j} \varphi_{j}^{K} R_{j}^{2} \\
\tilde{G}_{2}^{H}=C \sum_{K} \sum_{j} \varphi_{j}^{K} R_{j}^{2} m^{H K} \bar{\gamma}^{K} / \sum_{K} \sum_{j} \varphi_{j}^{K} R_{j}^{2} \\
\tilde{M}^{H}=\sum_{K} \sum_{j} \varphi_{j}^{K} R_{j}^{2} m^{H K} \gamma^{H K} / \sum_{K} \sum_{j} \varphi_{j}^{K} R_{j}^{2}
\end{array}\right.
$$

with $C=C^{\prime}\left(S_{i} / 4 \pi R_{i}^{2}\right)$ being a geometrical constant depending on the grain size distribution of the system and having the value around unity. By the condition of volume conservation during GG the constant $C$ can be easily determined. 


\section{DISCUSSIONS}

The process of grain growth is in principle the diffusion of GBs in a grain structure. Being different from the diffusion of an atom, which can be considered as a "null-dimensional" point, grain growth is the diffusion with "twodimensional" grain boundaries. Since all GBs construct an interconnected net spreading over the structure, the growth rate of a grain, i.e. the diffusion of the GBs of any grain must be connected with the nature of the whole grain structure, not only of its neighbouring grains. One recognizes that the partial diffusivities defined above are the averages of $m \gamma$ with the boundary area as weight factors, so that they are of rather "systematically integrated" nature. Moreover, the partial diffusivities $\tilde{G}_{1}^{H}$ and $\tilde{G}_{2}^{H}$ have a bearing on the radial boundary energy $\bar{\gamma}^{K}$ while $\tilde{M}^{H}$ on the "surrounding" boundary energy $\gamma^{H K}$ with the boundary mobility $m^{H K}$ as the same dynamic magnifictor. This can be easily understood since the driving force of a grain boundary is originated from the boundary curvature which is related with the equilibrium between the surrounding and radial boundaries along the lines or points where the boundaries are intersected.

For a better understanding of the new model the following interpretations of Eq. (33) can be given. $\tilde{M}^{H} / R_{i}$ is the effect socalled "shrinking-force" on the grain. It has always a negative sign and is inversely proportional to the size of the grain. If a grain were affected no other force, i.e. if the average specific boundary energy of the system were so small that it could be neglected in comparison with the specific boundary energy of the considered grain, the grain would remain in a spherical form with the boundary curvature equal to its radius. In this extreme case such a grain will always shrink away with the "growth" rate according to Eq. (33) and (34):

$$
\left(\frac{d R}{d t}\right)_{i}^{H} \simeq-2 \frac{M^{H}}{R^{i}} \quad(\text { when } \bar{\gamma} \simeq 0)
$$

One can easily see that this is equivalent to Eq. (10) since in the considered case $\rho_{1}=\rho_{2}=-R_{i}$ and $m \gamma=\tilde{M}^{H}$.

However, since the total grain volume of the structure must remain constant during GG, another force in the opposite direction of the shrinking-force must exist, which pulls the grain boundary outwards. This force is called "growingforce" and originates from the energy of grain boundaries besides the surrounding boundary of the considered grain itself. The growing-force is represented in Eq. (33) by two terms: $\tilde{G}_{1}^{H} / \bar{R}$ and $\tilde{G}_{2}^{H} / R_{i}$. The former is related to the grain boundary energy per volume of the system and is independent on size of the considered grain. The later is related to the "density" of the radial boundaries of the considered grain, since it is the radial boundary through which the growing-force affects on the grain. Because large grains have large (average) boundary section areas in the statistic sense, the radial boundary "density" of large grains is small, and thus the growing-force affecting on unit boundary is small. Hence the second growing-force term is inversely proportional to the grain size $R_{i}$.

It is well known that the mobility and specific energy of a large-angle GB are larger than those of a small-angle GB. The simulation by Eichelkraut (1988) shows that a large-angle boundary has a mobility up to 9 times as large as a 
small-angle boundary. The difference of the specific energy between different boundaries is much smaller than the difference in mobility, and in principle can not be larger than two times. Just because of this, the specific boundary energy is considered as a constant in many old GG models. Here we must point out, however, that in spite of its small difference the specific boundary energy has a rather large effect on the process of GG. Large grains in a structure can therefore grow only because the energy increase due to the increase of the surrounding boundary area during their growth is smaller than the energy decrease caused by the decrease of the radial boundary area. In the case of constant $\gamma$ the critical radius of GG, $R_{\mathrm{cr}}$, is then determined only by the grain size distribution and is a universal constant for the structure at a certain time. Grains larger than $\boldsymbol{R}_{\mathrm{cr}}$ will grow and others will shrink.

But this principle does not hold for a textured structure. To understand this one can see a 2-dimensional example shown in Figure 3 where two 8-sided grains have the same size but different orientation ( $A$ - and $B$-orientation respectively), and both grains are surrounded by other $A$-oriented grains. If $\gamma^{A B}=1.6 \gamma^{A A}$, the $A$ grain with the inner angle of $120^{\circ}$ has concave boundaries (Figure $3 a$ ) and the $B$ grain with the inner angle of

$$
2 \arccos \left(\frac{\gamma^{A A}}{2 \gamma^{A B}}\right)=144^{\circ}
$$

has convex boundaries (Figure $3 b$ ). Thus the $A$ grain will grow and the $B$ grain will shrink with time. Generally, in a 2-dimensional case, considering a $N$-sided regular (but with curved boundaries) grain with the surrounding boundary energy being $\gamma_{S}$ (in Figure $3 \mathrm{~b} \gamma_{S}=\gamma^{A B}$ ) and the radial boundary energy being $\gamma_{R}$ (in Figure $3 \mathrm{~b} \gamma_{R}=\gamma^{A A}$ ), since the boundary inner angle necessary for the equilibrium of the boundary tensions at the triple points is

$$
2 \arccos \left(\frac{\gamma R}{2 \gamma_{S}}\right)=\pi-2 \arcsin \left(\frac{\gamma_{R}}{\gamma_{S}}\right)
$$

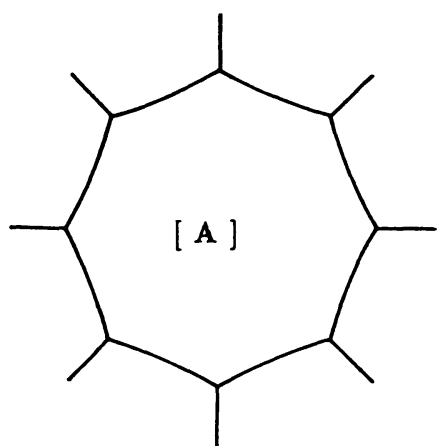

a. [A] grain surrounded by [A] grains

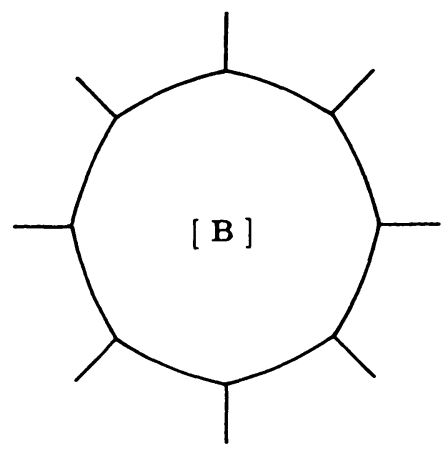

b. [B] grain surrounded by $[A]$ grains

Figure 3 An example to show the dependence of boundary curvatures on grain orientations. (a) the boundary energy is a constant, an 8 -sided grain will be concave and so that grow as indicated by $\mathrm{J}$. von Neumann, while (b) if the radial boundary energy is larger than the surrounding boundary energy, a grain with more than 6 sides can be also convex and shrink. 
and the inner angle for a $N$-sided regular grain with straight boundaries is $\pi-2 \pi / N$, one obtains the critical grain side:

$$
N_{\mathrm{cr}}=\pi / \arcsin \left(\frac{\gamma_{R}}{2 \gamma_{S}}\right)
$$

Grains with $N>N_{\mathrm{cr}}$ have concave boundaries and will grow while those with $N<N_{\text {cr }}$ will be convex and shrink. In textureless cases it is generally assumed that $\gamma_{R}=\gamma_{S}=\gamma$, so one has from Eq. (38) $N_{\mathrm{cr}}=6$ as being indicated by Neumann's law Eq. (1). However, in a textured structure grain boundary energy is anisotropic, the growth behaviour of any grain is therefore not only dependent on its geometric characters such as size and shape, but also strongly dependent on the orientations of itself as well as its neighbouring grains.

As an example the experimental results with $95 \%$ rolled, $300^{\circ} \mathrm{C}$ recystallized and subsequently $450^{\circ} \mathrm{C}$ annealed $\mathrm{Al}-3 \% \mathrm{Mg}$ pure binary alloy are given in Figure 4 for texture analysis and Figure 5 for the GG kinetics. The figures show that the process of GG can be divided into three stages. Stage I can be called "texture strengthening stage" because the volume percentage of the major component (cube texture) increases during this stage, although the average grain size of the minor component ( $Q$ and $R$ texture with Eulerian angles of $\left(65^{\circ}, 10^{\circ}, 0^{\circ}\right)$ and $\left(60^{\circ}, 33^{\circ}, 55^{\circ}\right)$ respectively), $\bar{R}^{Q+Q}$, increases much faster than that of the cube texture, $\bar{R}^{\text {cube }}$. During stage II the volume of cube component decreases dramatically from about $95 \%$ to null, so that it is called "texture change stage." And in stage III only one texture component i.e. $Q+R$ component remains in the system. It is therefore called "one component stage."

To understand the nature of the experimental result in the view of the new model, the partial diffusivities of both components are calculated according to Eq. (34) with $\varphi_{j}^{K}$ being obtained experimentally and $m^{H K}$ and $\gamma^{H K}$ being given by the computer simulations (Zhao, 1990), and plotted in Figure 6 vs. annealing time.

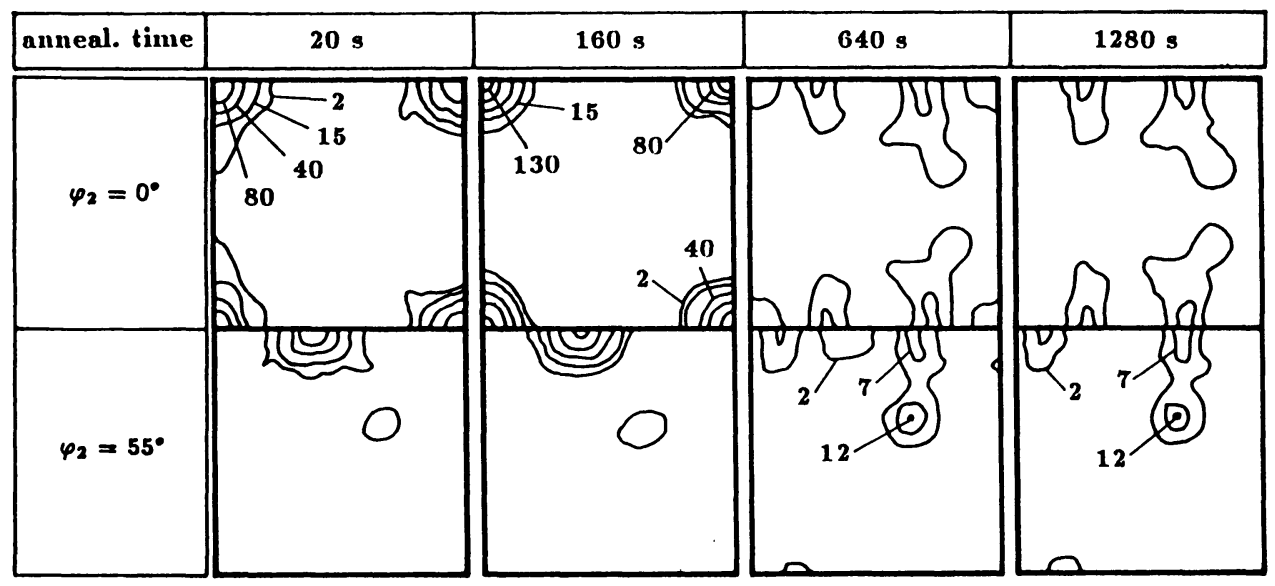

Figure 4 The texture development during annealing at $450^{\circ} \mathrm{C}$ of $\mathrm{Al}-3 \% \mathrm{Mg}$ samples pre-treated with $95 \%$ rolling and $300^{\circ} \mathrm{C}$ recrystallization. The textures are given in the form of true ODFs with the sections of $\varphi_{2}=0^{\circ}$ and $55^{\circ}$. 

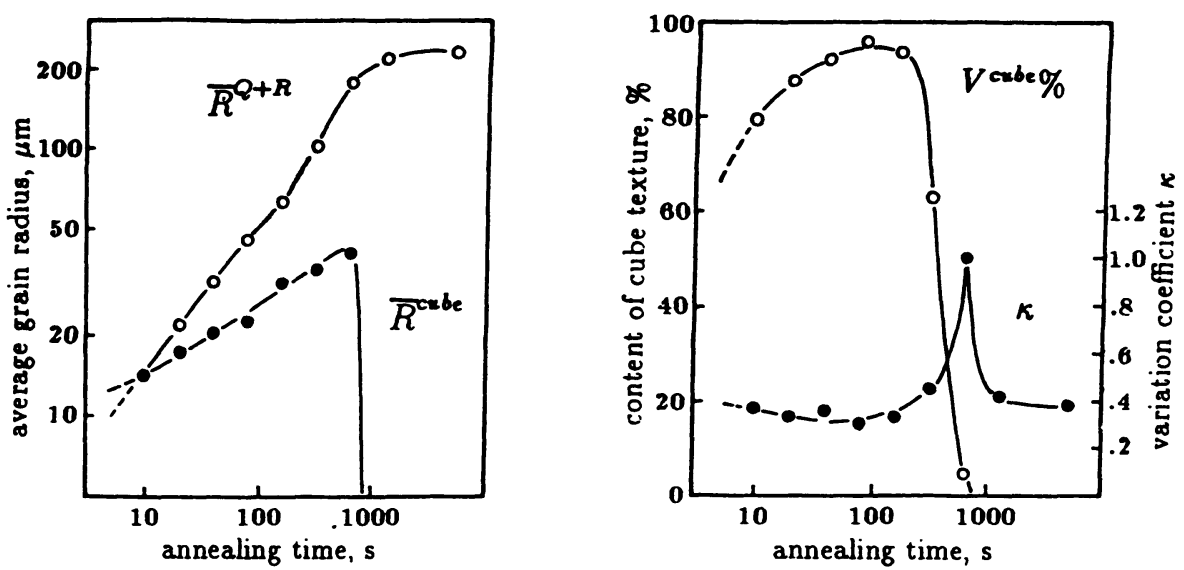

Figure 5 The grain growth kinetics of $\mathrm{Al}-3 \% \mathrm{Mg}$ samples during annealing at $450^{\circ} \mathrm{C}$.

At the beginning of the annealing the grain size of cube texture is similar to that of $Q+R$ component. As the major component, cube grains have statistically boundaries with low mobility and low energy, which means first that the boundary migration and thus the grain growth rate for cube component will be slow and second that the number of cube grains which can grow will be large since they have low energy surrounding boundaries. In Figure 6 one sees that the partial diffusivities of cube component have small but similar values, which indicates according to Eq. (33) that for cube component grains with size slightly over $\bar{R}$ are large enough to grow. In other side for $Q$ and $R$ oriented grains, from Figure 6 one sees that all three partial diffusivities have high values, which is contributed mainly by the high mobility of the large angle GBs surrounded $Q+R$ grains. But this does not mean that all $Q+R$ grains have high growing rate. Because of the high surrounding boundary energy, $Q+R$ grains are affected by very strong shrinking forces, as one sees in Figure 6 that $\tilde{M}^{Q+R}$ is remarkably higher than $\tilde{G}_{1}^{Q+R}$ and $\tilde{G}_{2}^{Q+R}$. Therefore according to $\mathrm{Eq}$. (33), for $Q+R$ component only a few grains with sizes much larger than $\bar{R}$ can grow during GG, while the most $Q+R$ grains will shrink although they are probably larger than the growing cube grains.

It can be then concluded that due to the effect of texture, at the beginning of annealing, a lot of cube grains grow but slowly, and a few large $Q+R$ grains grow fast but most $Q+R$ grains will shrink rapidly. Therefore in stage I of GG, the volume of cube component goes up but the size of cube grains increases very slowly, and $Q+R$ component decreases in volume but increases very fast in average grain size, which is originated not only by the fast growing rate of the large $Q+R$ grains but also by the vanishing of the (relative) small $Q+R$ grains. As cube texture strengthens the effect of texture increases, so one sees from Figure 6 that the difference on partial diffusivities between cube and $Q+R$ components is enlarged during stage I of GG.

At the end of stage $I, \bar{R}^{Q+R}$ is about four times as $\bar{R}^{\text {cube }}$. Due to the high boundary mobility a few very large $Q+R$ grains grow very fast at the cost of the small cube grains, which leads to a strong secondary recrystallization in stage II 


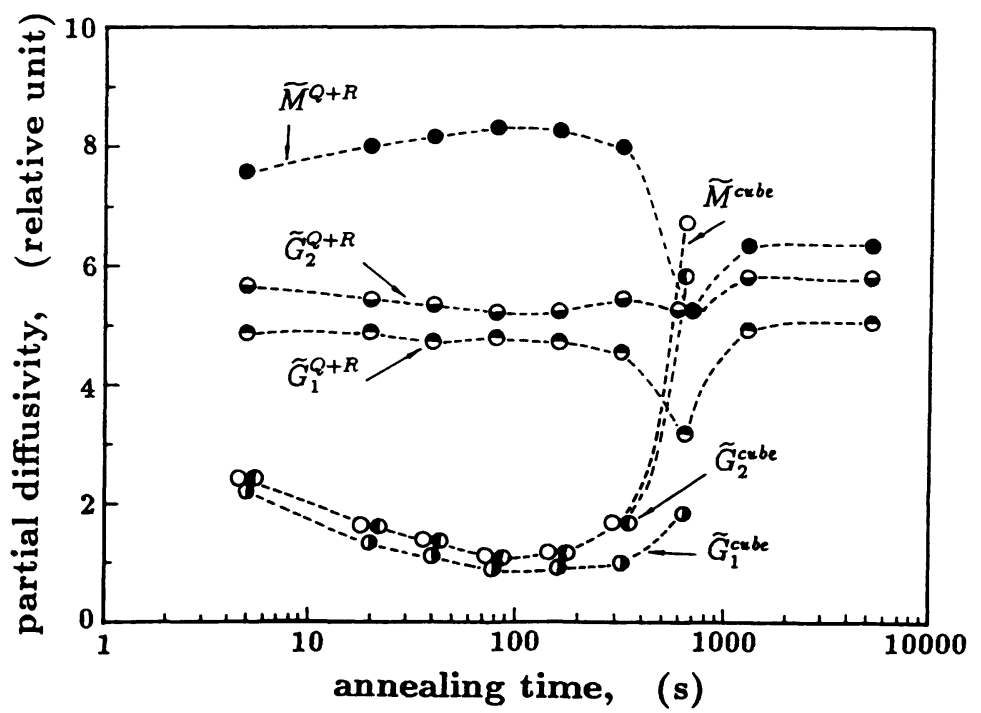

Figure 6 Relative partial diffusivities of both components of $\mathrm{Al}-3 \% \mathrm{Mg}$ samples during annealing at $450^{\circ} \mathrm{C}$.

with two significant features (see Figure 5b): a texture change with the result of the vanishing of the cube texture and a high peak in the $K$-curve when the number of the small cube grains approaches to the number of the large $Q+R$ oriented grains. The change of the partial diffusivities during stage II indicates that the cube GBs will move faster than before but they move practically all inwards leading to the shrinkage of cube grains. In the other side, for $Q+R$ component the descent of $\tilde{M}^{Q+R}$ implies that since more and more growing grains meet them each other, their growth rates are largely reduced as one sees from Figure 5b.

After the texture change stage only a few very large $Q+R$ grains remain in the system. In stage III because of the large grain sizes and the low boundary mobility, the grain growth rate is very small.

As to the phenomenon of texture strengthening during GG, one can see from the above discussion that it is caused directly by the anisotropy of grain boundary energy and is an important cause of the texture induced secondary recrystallization, since the texture strengthening enlarges the difference on partial diffusivities between both texture components. This argument is contradictory to the common sense that the volume percentage of the minor component always increases during GG in a textured system (Eichelkraut, Abbruzzese and Lücke 1988; Eichelkraut, 1988). However it is a logical and reasonable inference from our model, and has been also found experimentally, e.g. in $\mathrm{Al}-\mathrm{Mg}$ and $\mathrm{Al}-\mathrm{Mn}$ alloys (Zhao, 1990; Heckelmann et al., 1990).

\section{CONCLUSIONS}

The process of grain growth in the presence of textures has been statistically analysed based on the consideration of total free energy change of the system 
during the migration of grain boundaries. The presently developed grain growth model indicates that the difference of specific grain boundary energy plays a rather important roll in the process of texture controlled grain growth. A quantitative analysis shows that the large difference in specific boundary energy caused by the presence of strong textures is a hindrance effect on the grain growth of the minor texture component since its critical radius is much larger than that of the major component. Thus texture strengthening during grain growth can occur when the texture after the primary recrystallization is strong enough, which in turn, leads to the secondary recrystallization with a drastic texture change during the subsequent annealing.

\section{Acknowledgement}

The author is very thankful to Prof. Dr. K. Lücke for many helpful discussions. The work is financially supported by the National Natural Science Foundation of China.

\section{References}

Abbruzzese, G. and Lücke, K. (1986). "A Theory of Texture Controlled Grain Growth-I. Derviation and General Discussion of the Model." Acta Met. 34, 905-914.

Abbruzzese, G., Lücke, K. and Eichelkraut, H. (1988). "Grain growth in the presence of textures-theoretical approach and comparison to experiments." In Proc. 8th Intern. Conf. on Textures of Materials (ICOTOM 8). AIME, Santa Fe, Pennsylvania, USA. pp. 693-705.

Bunge, H. J. and Dahlem, E. (1987). "Steady State Grain Growth in Textured Materials." In: Theoretical Methods of Texture Analysis. Edited by H. J. Bunge. DGM Informationsgesellschaft, Oberursel, pp. 301-324.

Bunge, H. J. and Dahlem-Klein, E. (1988). "Model Calculation of Grain Growth in Textured Materials." In Proc. 8th Intern. Conf. on Textures of Materials (ICOTOM 8). AIME, Santa Fe, Pennsylvania, USA. pp. 705-710.

Eichelkraut, H. (1988). "Theoretische und experimentelle Untersuchungen zum Kornwachstum in Metallen in Gegenwart von Texturen und Partikeln". Doctor Thesis. RWTH Aachen, Aachen, FRG.

Eichelkraut, H., Abbruzzese, G. and Lücke, K. (1988). "A Theory of Texture Controlled Grain Growth-II. Numerical and Analytical Treatment of Grain Growth in the Presence of two Texture Components." Acta Met. 36, 55-68.

Heckelmann, I., Zhao, X. B., Lücke, K. and Abbruzzese, G. (1990). "Texture Development During Grain Growth." In Proc. 9th Intern. Conf. on Textures on Materials (ICOTOM 9). Avignon, France.

Hillert, H. (1965). "On the Theory of Normal and Abnormal Grain Growth." Acta Met. 13, 227-238.

Mullins, W. W. and Viñals, J. (1989). "Self-Similarity and Growth Kinetics Driven by Surface Free Energy Reduction." Acta Met. 37, 991-997.

Mullins, W. W. (1986). "The statistical self-similarity hypothesis in grain growth and particle coarsening." J. Appl. Phys. 59, 1341-1349.

Von Neumann, J. (1952). Discussion on "Shape of Metal Grains." In: Metal Interfaces, pp. 108-110, ASM, Cleveland, Ohio, USA.

Zhao, X. B. (1990). "A Study on the Grain Size Distribution and Texture Controlled Grain Growth in Cold Rolled Sheet Metals." Doctor Thesis (in Chinese). Zhejiang University, Hangzhou, China. 